\title{
Spike timing precision and neural error correction: local behavior
}

\author{
Michael Stiber \\ Computing \& Software Systems \\ University of Washington \\ Bothell, WA, 98011-8246 USA \\ stiberlu.washington.edu
}

October 25,2018

\begin{abstract}
The effects of spike timing precision and dynamical behavior on error correction in spiking neurons were investigated. Stationary discharges — phase locked, quasiperiodic, or chaotic - were induced in a simulated neuron by presenting pacemaker presynaptic spike trains across a model of a prototypical inhibitory synapse. Reduced timing precision was modeled by jittering presynaptic spike times. Aftereffects of errors — in this communication, missed presynaptic spikes — were determined by comparing postsynaptic spike times between simulations identical except for the presence or absence of errors. Results show that the effects of an error vary greatly depending on the ongoing dynamical behavior. In the case of phase lockings, a high degree of presynaptic spike timing precision can provide significantly faster error recovery. For non-locked behaviors, isolated missed spikes can have little or no discernible aftereffects (or even serve to paradoxically reduce uncertainty in postsynaptic spike timing), regardless of presynaptic imprecision. This suggests two possible categories of error correction: high-precision locking with rapid recovery and low-precision non-locked with error immunity.
\end{abstract}

\section{Introduction}

This work is concerned with the effects of spike timing precision and dynamical behavior on the recovery of ongoing neural discharges after a perturbation, or an error. The concepts of "precision" and "error" are of course intimately linked. Determining how information is transmitted among neurons is an essential issue in computational neuroscience, with a spectrum of possibilities, ranging from overall average firing rates to timing of individual spikes (Dan et al., 1998; 
Lestienne, 2001). Central to the question of where a presynaptic neuron's code lies in this spectrum is the precision of spike timing necessary to elicit a particular response from a postsynaptic neuron. This follows logically, as two different presynaptic discharges which produce the same postsynaptic response have achieved the same functional result, and thus can be said to have carried the same message.

The present paper addresses this issue directly by comparing recovery from errors in a spike train for two levels of spike timing precision. The goal is to test the feasibility of the idea that extra spike timing precision (in the sense that it is more than the minimum necessary to transmit a given amount of information) can be used for error correction. The errors that are used are missed spikes, based on the observed apparent unreliability of synaptic transmission (Katz, 1966, Allen and Stevens, 1994).

Examination of the effects of noise and jitter in spike trains on neural behavior goes back decades (Segundo et al., 1963; Segundo et al., 1968; Bryant et al., 1973; Segundo et al., 1976; Kohn et al., 1981 , Kohn and Segundo, 1983; Segundo et al., 1994b). Briefly, the larger the number of input synapses (the smaller the effect of each presynaptic spike) and the smaller the correlation among them, the more their aggregate postsynaptic effect was like a DC bias. Greater correlations and fewer, stronger synapses operated more like single, strong synapses, producing reliably repeatable responses in the postsynaptic cell. The effect of increasing noise on ongoing dynamical behavior in pacemakers was observed as steadily narrowing the input rate domains for phase locked behaviors, abolishing those with narrower domains before those with broader ones. Consequently, periodic behaviors with broader domains in parameter space were more stable in the presence of noise. Later work showed this effect in rat spinal interneurons (Beierholm et al., 2001), and presented similar results for $1: 1$ alternation (the non-pacemaker counterpart of phase locking) with quasiperiodic driving (Tiesinga, 2002).

Analysis of networks showed that coupling large numbers of cells could lead to lower output variability in the face of input jitter (Maršálek et al., 1997, Tiesinga and Sejnowski, 2001). Additionally, periodic, phase locked dynamics were noted to be optimal for reduction of output jitter (the exception to this being near bifurcation boundaries, where input jitter could occasionally abolish locking) in network models (Tiesinga and Sejnowski, 2001).

This paper investigates how timing precision in a presynaptic train interacts with the destruction of information caused by a single neuron's internal dynamics. It extends previous work by evaluating both periodic and aperiodic (quasiperiodic and chaotic) postsynaptic dynamics, as well as the effects of proximity to bifurcations bounding periodic behaviors. Additionally, rather than simply examining the amount of perturbation produced by presynaptic errors, it also analyzes the time course of recovery. The ongoing presynaptic spike trains used here were stationary ones, with and without jitter, that induced a range of dynamical behaviors in the postsynaptic cell. Error recovery was measured by the time course of return of the postsynaptic cell to its stationary behavior. This was determined by comparing pairs of simulations that were identical except for the presence of errors, so that after an error, the spike train of the postsynaptic cell could be compared to that of one that didn't see the error. Pairs of simulations were repeated for inputs with and without jitter, and error recovery was then compared in those two cases. 


\section{Methods}

A well-characterized physiological model (see Appendix) of the crayfish slowly adapting stretch receptor organ, or SAO, was used. In the living preparation, the SAO produces spike trains with rate broadly proportionate to muscle stretch; at constant stretch, it produces pacemaker spike trains. It includes the recognized prototype of a moderately-powerful, GABAergic inhibitory synapse, and thus has been used as a living model to explore synaptic coding across inhibitory synapses in general. The model has been used previously to explain the dynamics behind "paradoxical accelerations" observed in the living preparation (Segundo, 1979, Buño et al., 1987) in response to pacemaker inhibitory PSPs (Segundo et al., 1991a; Segundo et al., 1991b), as well as the SAO's responses to nonstationary spike trains (Segundo et al., 1994a; Segundo et al., 1995b; Segundo et al., 1995a, Stiber et al., 1997; Segundo et al., 1998).

Presynaptic spike train timing generation and analysis was performed in MATLAB (The MathWorks, Inc.). Simulation was performed using custom $\mathrm{C}$ code and the ODEPACK differential equation integration library (Hindmarsh, 1983). Both pre- and postsynaptic spike trains were assimilated to point processes (Cox and Isham, 1980) and all analysis was based on inter-event intervals.

In the absence of presynaptic input, the SAO and its model behave as pacemakers, producing action potentials with an almost invariant interspike interval, $N$ : its natural interval. In the presence of pacemaker presynaptic trains, both exhibit a variety of dynamical behaviors, including phase lockings, quasiperiodicities, and chaos, with characteristic dependencies on the presynaptic rate (Segundo et al., 1991a, Segundo et al., 1991b; Stiber and Segundo, 1993; Stiber et al., 1996).

The current work began by building on previous works' survey of the dynamical behaviors exhibited by the model. Approximately 10,000 simulations were performed, varying both presynaptic rate $(N / I, 1 / I$ being the presynaptic rate for each simulation) and IPSP strength $(P)$ within biologically plausible limits. This was used to produce an Arnol'd map, or two-dimensional bifurcation diagram, which categorized each simulation's dynamical behavior within the $2 \mathrm{D}(N / I, P)$ plane. Three values of $P$ were then chosen for more detailed examination. These were dubbed "weak", "moderate", and "strong" IPSPs, based on the ratio of locked (periodic) to non-locked (aperiodic) behaviors they engendered $(\leq 20 \%, \approx 50 \%$, and $\approx 100 \%$, respectively). For the current investigation, a moderate value of IPSP strength was chosen.

A range of normalized presynaptic rates $0.2 \leq N / I \leq 2.0$ was then explored using one-dimensional bifurcation diagrams. Here, such diagrams were constructed by plotting postsynaptic spike phase (cross interval from a postsynaptic spike back to the most recent presynaptic spike) versus $N / I$. These allowed easy determination of the distribution and organization of qualitatively different dynamical behaviors along the presynaptic rate scale (types of behaviors, behavior ranges, and bifurcation locations). Each bifurcation diagram involved a set of 90-200 simulations, with diagrams produced for pacemaker and jittered pacemaker presynaptic spike trains. Values of jitter ( $w$, see below) ranging from $0.01 I$ to $0.1 I( \pm 1 \%- \pm 10 \%)$ were examined; their bifurcation diagrams were similar overall. Results for $\pm 1 \%$ jitter are presented here. This was viewed as a stricter test of the hypothesis (that high precision inputs would result in faster recovery from errors than low precision, and thus the extra precision could be viewed as a type of redundant, error-correcting code) than larger values of $w$, as larger values would certainly result in the model's state being perturbed farther from the stationary attractors (and thus it would be a much shorter distance, therefore faster, 


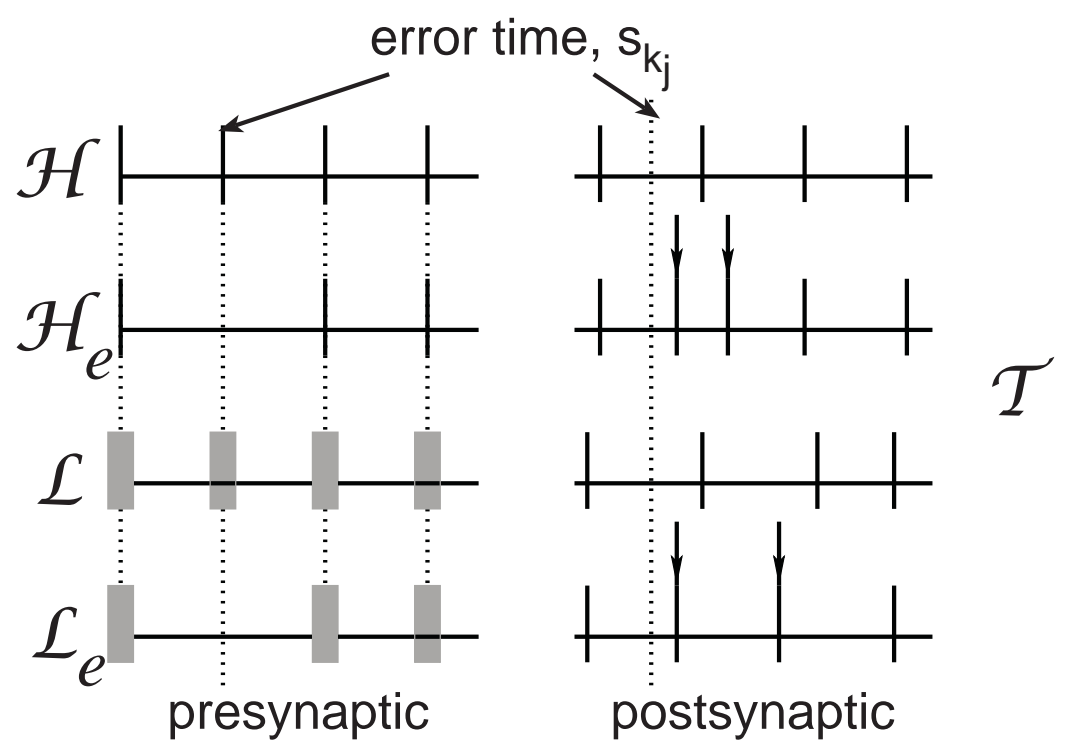

Figure 1: Simulation methods. Pairs of high $\left(\mathscr{H}, \mathscr{H}_{e}\right)$ or low $\left(\mathscr{L}, \mathscr{L}_{e}\right)$ precision spike trains were applied via the simulated inhibitory fiber (IF). Postsynaptic spike times $(\mathscr{T})$ were recorded for analysis.

for the model to return to its attractor for high-precision input). Work on evaluating, in detail, the effects of varying level of jitter on error recovery are ongoing (Stiber and Holderman, 2004b).

Four sets of 90-100 simulations used to produce bifurcation diagrams (high precision, low precision, and each with errors, see below) then served as a database describing error responses of the model. This communication focuses on the details of the model's recovery after an error and the effects of presynaptic spike timing precision on that recovery, and so a few illustrative behaviors were selected. The behaviors chosen are archetypical of the full range of dynamical behavior types exhibited by driven nonlinear oscillators: $1: 1$ locking (period 1), $1: 2$ locking (period 2), $2: 3$ locking (period 3, the longest periodic behavior for this model with significant extent along the $N / I$ scale), two quasiperiodicities (low $-N / I<1.0-$ and high $-N / I>1.0$ - rate), and chaos. An additional behavior was chosen to illustrate the effects of proximity to a bifurcation. A more complete examination of the global behavior of error correction (e.g., two and three dimensional bifurcation structure) is under way (Stiber and Holderman, 2004a).

For each example behavior, simulations involved generating two pairs of almost identical presynaptic spike trains, as schematized in Figure 1 For each set of simulations, a reference, high precision train, $\mathscr{H}$, with spike times $\left\{s_{1}, s_{2}, \ldots, s_{n}\right\}$, was first generated. As this was a pacemaker train, all interspike intervals were identical $\left(\forall k, I=I_{k}=s_{k}-s_{k-1}\right) . I$ was the independent variable, chosen to elicit the example dynamical behaviors from the model. An error was defined to be complete failure of synaptic transmission, and so a set of spike numbers, $\mathscr{K}=\left\{k_{1}, k_{2}, \ldots, k_{m}\right\}$, was generated randomly such that all errors were separated by at least $15 \mathrm{~s}$ (to allow the neuron to return to its stationary behavior before each error). Presynaptic spikes at times $\mathscr{H}_{\mathscr{K}}=\left\{s_{k_{1}}, s_{k_{2}}, \ldots, s_{k_{m}}\right\}$ were eliminated from $\mathscr{H}$ to produce a high precision erroneous train, $\mathscr{H}_{e}=\mathscr{H}-\mathscr{H}_{\mathscr{K}}$.

A low precision counterpart of $\mathscr{H}$ was generated by jittering each spike $s_{k} \in \mathscr{H}$ by a value $u_{k}$ 


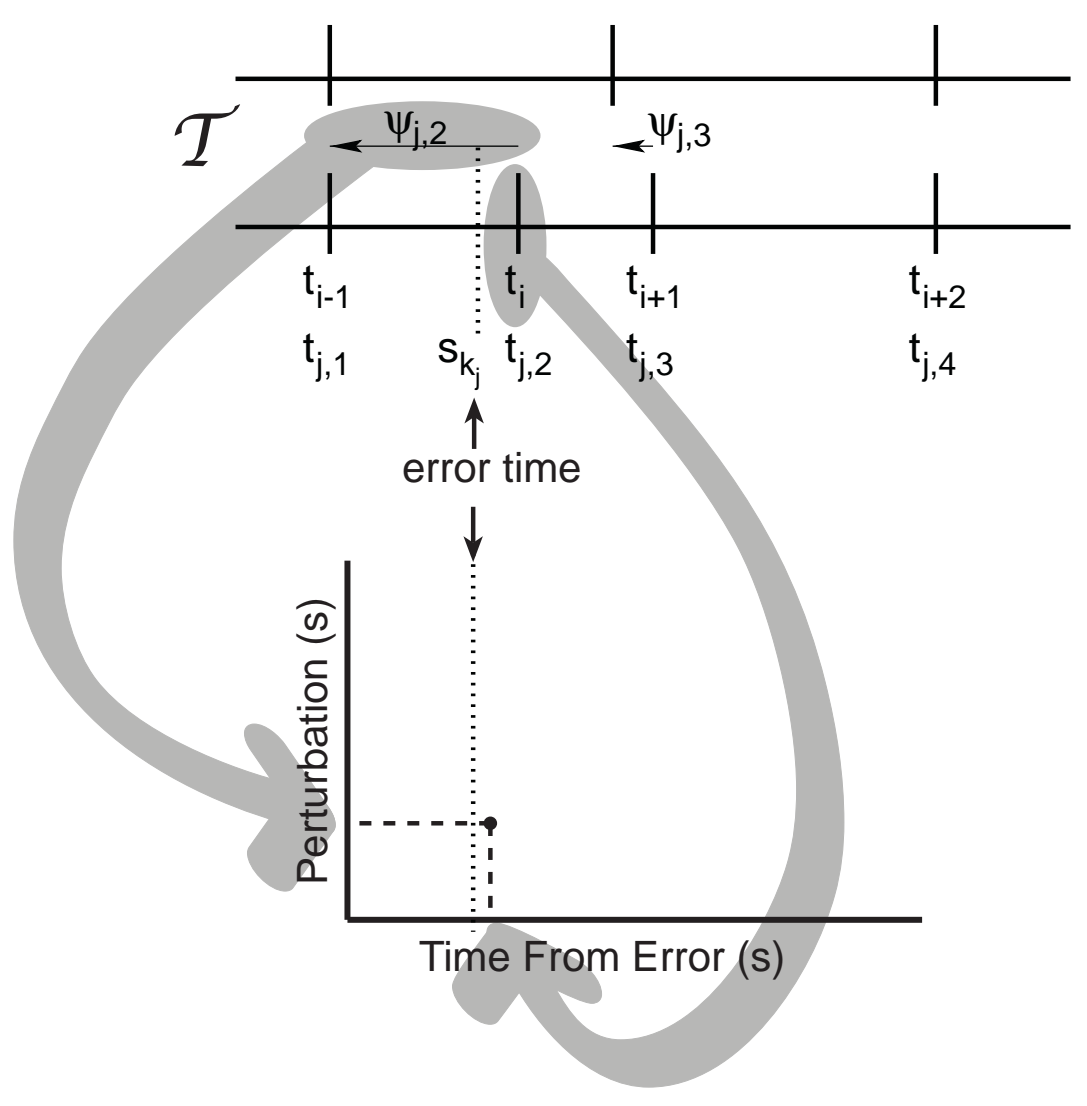

Figure 2: Perturbation graph generation. The displacement of a postsynaptic spike after an error (bottom train) from the time of the corresponding spike in the situation where there was no error (top train) is plotted versus time since the error.

taken from a uniform distribution with range $\pm w I$. In the current communication, a small value of $w(w=0.01,2 \%$ jitter $)$ was used.

The result of applying this jitter was a low precision train $\mathscr{L}=\left\{s_{1}+u_{1}, s_{2}+u_{2}, \ldots, s_{n}+u_{n}\right\}$. A low precision erroneous train, $\mathscr{L}_{e}=\mathscr{L}-\mathscr{L}_{\mathscr{K}}$, was then produced by eliminating the spikes numbered $\mathscr{K}$ at times $\mathscr{L}_{\mathscr{K}}=\left\{s_{k_{1}}+u_{k_{1}}, s_{k_{2}}+u_{k_{2}}, \ldots, s_{k_{m}}+u_{k_{m}}\right\}$. Subsequent analysis involved pooling timing data for postsynaptic spikes near errors in these sets of four simulations. As a double-check, multiple simulations were run for each set of simulation parameters (with different realizations of $\left\{u_{k}\right\}$ ) and analysis performed on the pooled data from all; as might be expected, there was no difference in results for $m$ errors taken from $n$ simulations versus $m$ errors taken from a single simulation.

As Figure 2illustrates, the first step of analysis was based on comparing times of postsynaptic spikes $\mathscr{T}=\left\{\ldots, t_{i-1}, t_{i}, \ldots\right\}$ just before and after the error times for the erroneous and error-free cases. For each error $k_{j} \in \mathscr{K}, j=1,2, \ldots, m$, the set of $c$ postsynaptic spikes just before and after were selected (here, 5 spikes before and 25,30 , or 45 spikes after - depending on the rate of production of postsynaptic spikes and the recovery time - and thus $c$ was 30,35, or 50). These spike times can be renamed relative to the error number $j$ as $\left\{t_{j, 1}, t_{j, 2}, \ldots, t_{j, c}\right\}$ (bottom train in 


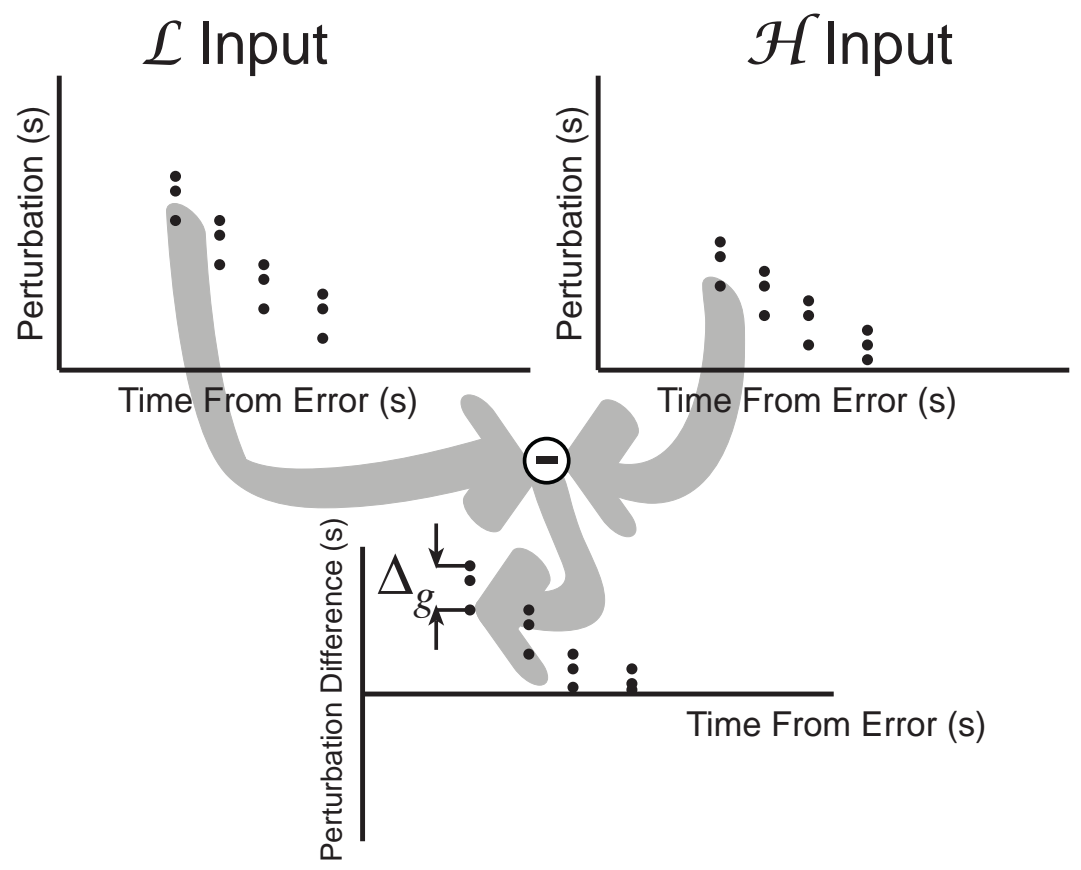

Figure 3: Recovery plot construction. A pair of corresponding high and low precision perturbation graphs (top) are subtracted to produce perturbation differences; the recovery plot is the plot of the range of differences, $\Delta_{g}$.

figure). For each postsynaptic spike in the erroneous case, $\left\{t_{j, g}\right\}, j=1,2, \ldots, m, g=1,2, \ldots, c$, the cross interval back to the most recent postsynaptic spike in the error-free case (top train in figure), $\psi_{j, g}$, was computed. As the simulations involve an inhibitory synapse, the number of postsynaptic spikes in the erroneous case was always greater than or equal to the number in the error-free case; this was the reason for using the former spikes as the reference for computing $\psi_{j, g}$. This was done for each pair of high and low precision input simulations to yield $\psi_{j, g}^{\mathscr{H}}$ and $\psi_{j, g}^{\mathscr{L}}$, respectively. Thus, $\psi_{j, g}^{\mathscr{H}}$ and $\psi_{j, g}^{\mathscr{L}}$ measure the shift, or perturbation, of each postsynaptic spike from the time it would have occurred if there had been no error. The graph of $\left(t_{j, g}-s_{k_{j}}, \psi_{j, g}^{\mathscr{H}}\right)$ or $\left(t_{j, g}-s_{k_{j}}-u_{k_{j}}, \psi_{j, g}^{\mathscr{L}}\right)$, such as in Figure 6 (A), was termed a perturbation graph.

In every case, the perturbation graph compares a simulation (either high or low precision) with errors to the identical (canonical) one without errors. Therefore, any scatter of points is due to either the simulation accuracy (which, for lockings at least, can be judged by the points before the error time) or the presence of errors themselves. Jitter, in and of itself, did not produce any scatter because each presynaptic spike in both simulations occurred at identical times in identical simulations (neglecting errors). Perturbation graphs show only the effects of errors and possible interaction of errors with the presence of jitter.

The high and low precision perturbations, $\psi_{j, g}^{\mathscr{H}}$ and $\psi_{j, g}^{\mathscr{L}}$, were compared in recovery plots. Figure 3 illustrates how such plots were generated. For each pair of cross intervals, the difference, $\delta_{j, g}=\psi_{j, g}^{\mathscr{L}}-\psi_{j, g}^{\mathscr{H}}$, was computed. Because of the variation in the individual error responses in the 
low precision case (the scatter of points in the perturbation plot), $\boldsymbol{\delta}_{j, g}$ typically took on a range of values for each $g$. Rather than examine the average difference, the decision was made to compute the range of differences, $\Delta_{g}=\max _{j}\left(\delta_{j, g}\right)-\min _{j}\left(\delta_{j, g}\right)$. A difference $\delta_{j, g}=0$ indicated that the high and low precision perturbations, for that particular error $j$ and postsynaptic spike $g$, were identical. A range $\Delta_{g}=0$ indicated that, for all errors, the perturbation of postsynaptic spike $g$ was the same for high and low precision - that low precision input did not have an effect on the perturbation. Thus, a range $\Delta_{g}>0$ indicated that some low precision perturbations differed from high precision ones. In other words, because these were simulations, none of the postsynaptic spikes were considered outliers. As an alternative, the variance of $\delta_{j, g}$ (for each value of $g$ ) could have been used; in practice, it produced equivalent results. So, a recovery plot such as in Figure 4 is a graph of $\Delta_{g}$ versus the mean offset $e_{g}=\overline{t_{j, g}-s_{k_{j}}}$, for each value of $g=1,2, \ldots, c$. It collapses the $g^{\text {th }}$ set of perturbations to a single point and compare high and low precision perturbations, showing the time course by which the worst low precision error responses approached those for high precision.

The primary motivations behind the use of recovery plots were easier analysis of the time course of recovery than perturbation graphs and elimination of the need to compare pairs of perturbation plots by eye to evaluate the effects of low versus high precision. Assuming the erroneous simulation fully recovered before each error, the values of $\Delta_{g}$ for $e_{g}<0$ can also serve to test whether low precision responses were still significantly perturbed. Comparing $\Delta_{g}$ for $e_{g}<0$ for different simulation parameters allows one to judge whether the simulation was recovered.

To characterize the ongoing dynamical behavior exhibited by the SAO, cross intervals between the pre- and postsynaptic times, $\mathscr{H}$ and $\mathscr{T}$, respectively, were used. Since $\mathscr{H}$ was a pacemaker process, the cross intervals $\phi_{i}=t_{i}-s_{k}$ from postsynaptic spikes back to most recent preceding presynaptic spikes were termed phases (Winfree, 1980; Glass and Mackey, 1988). The phase return map, plotting $\left(\phi_{i}, \phi_{i+q}\right)$, trivially indicates $p: q$ locking since $\phi_{i}=\phi_{i+q}$. Quasiperiodic responses produce a 1D curve without a maximum for $q=1$, while chaotic behaviors have return maps with maxima or that are not one-dimensional curves (Abraham and Shaw, 1984, Rapp et al., 1985; Ruelle, 1989). For brevity's sake, return maps are omitted from the Results section for phase lockings.

\section{Results}

In this section, error recovery during locked, quasiperiodic, and chaotic responses is considered. A final subsection examines the effect of proximity to a bifurcation boundary on error recovery.

\subsection{Phase Lockings}

In previous work on both the SAO and this model, phase locked responses were found to be the most robust in the presence of nonstationary presynaptic spike trains, with $1: 1$ locking being the most robust among lockings (Segundo et al., 1993; Segundo et al., 1994a; Segundo et al., 1995b; Segundo et al., 1995a, Segundo et al., 1998; Stiber et al., 1997). This has also been noted in other preparations and simulations (Beierholm et al., 2001; Tiesinga and Sejnowski, 2001; Tiesinga, 2002). 

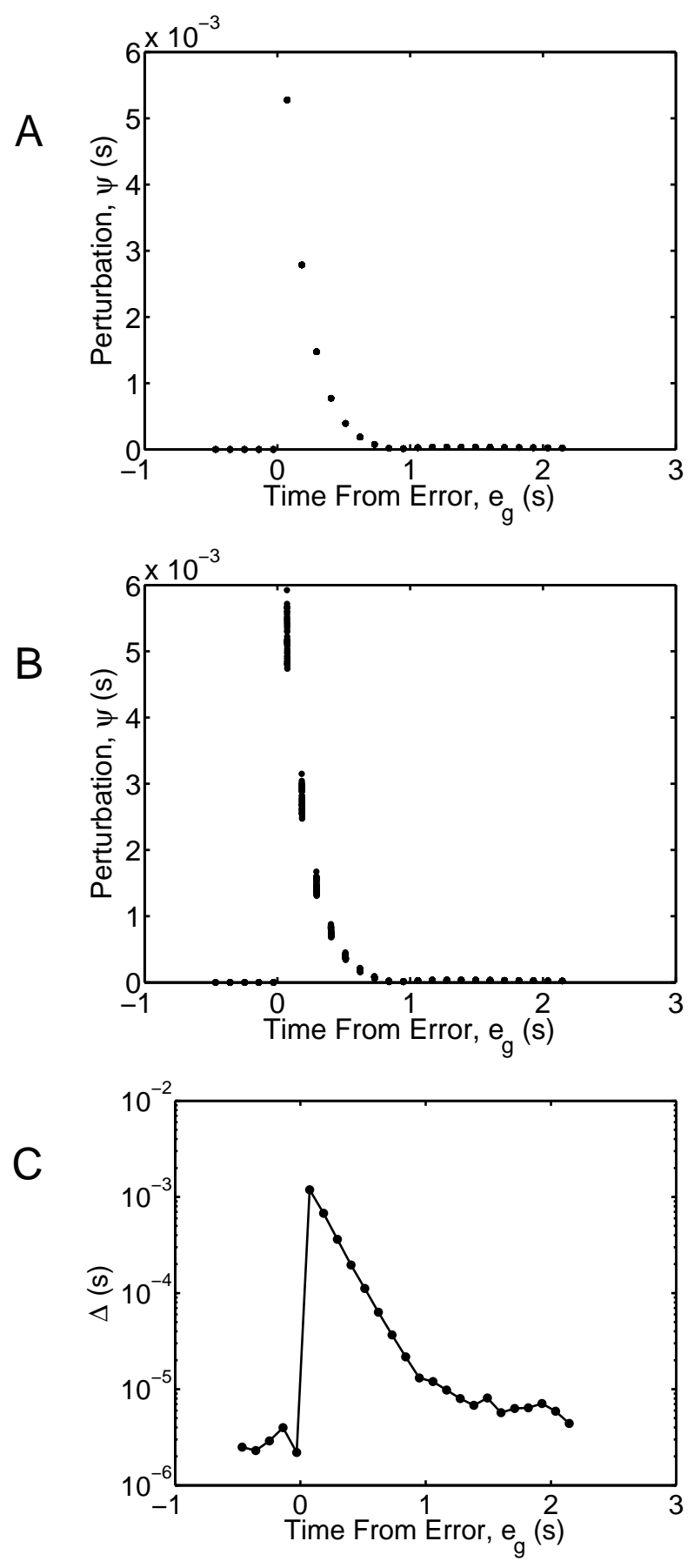

Figure 4: Results for center of $1: 1$ locking $(I=108.78 \mathrm{~ms}, N / I=0.96)$. High precision perturbation graph (A) shows identical responses to all errors; low precision (B) shows range of responses. Recovery plot $(\mathrm{C})$ shows long-duration recovery. ( $m=49$ errors, $c=25$ spikes/error) 

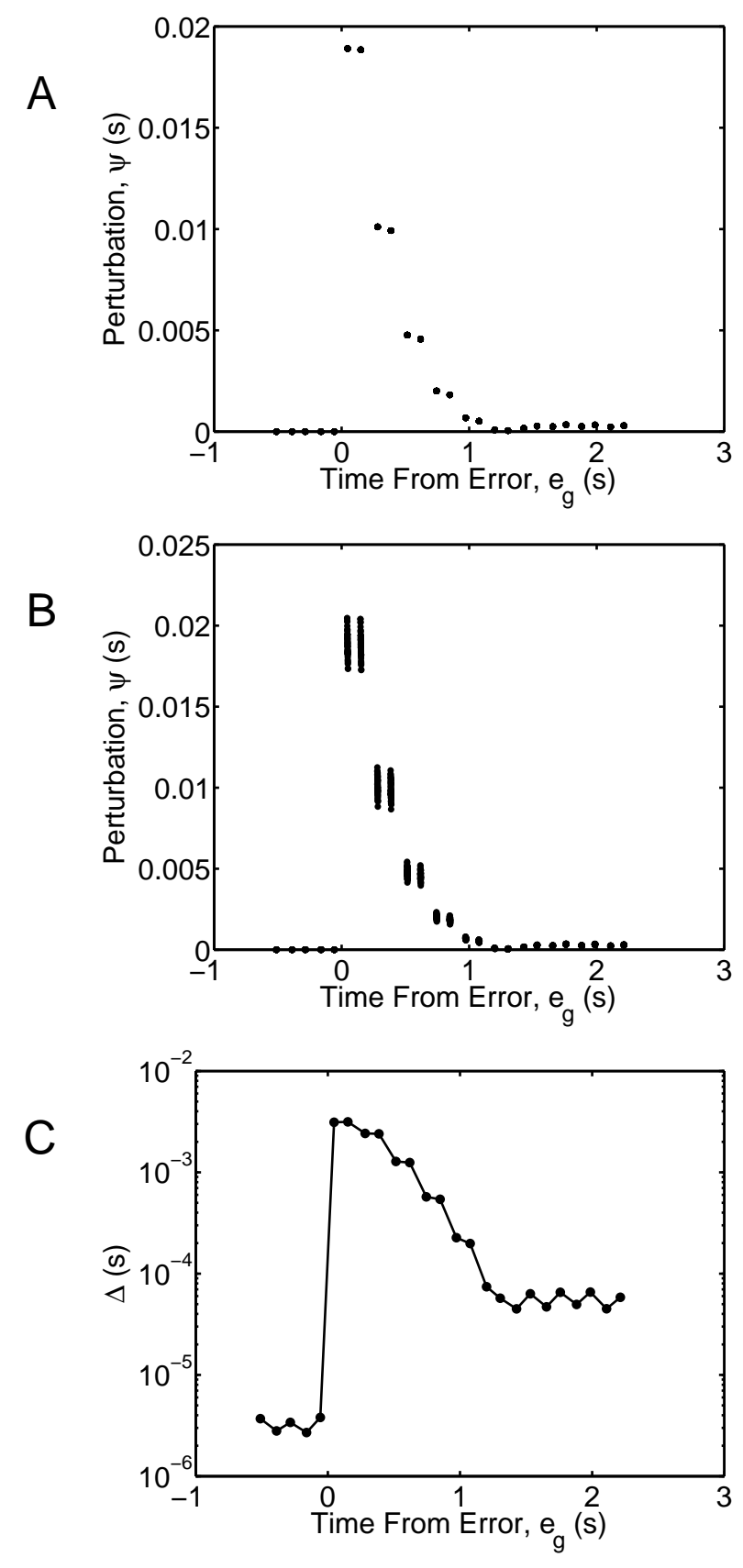

Figure 5: Results for $1: 2$ locking $(I=227.01 \mathrm{~ms}, N / I=0.46)$. Perturbation graphs for high (A) and low (B) precision input and recovery plot (C) all carry mark of the two categories of phases. ( $m=49$ errors, $c=25$ spikes/error)

Thus, analysis began with these behaviors in the expectation that they would exhibit the greatest immunity to low precision jitter and the fastest error recovery times (Stiber, 2003).

Figure 4 presents typical results for $1: 1$ locking. For high precision input, the response to every 
error was identical (A), with a maximum perturbation of approximately $\psi_{j, g}^{\mathscr{H}}=5.25 \mathrm{~ms}$. For low precision (B), the maximum perturbation was $\psi_{j, g}^{\mathscr{L}} \approx 6 \mathrm{~ms}$, with up to a $1.2 \mathrm{~ms}$ range for any single value of $g$. An exponential fit for the points in the recovery plot $(\mathrm{C})$ in the range $0.4 \leq e_{g} \leq 0.9$ yielded a recovery time constant of $\tau=0.20 \mathrm{~s}(\tau / I=1.84)$, with a slower recovery proceeding thereafter. This is the rate at which the "worst" low precision error responses approached that for high precision. Low precision responses were still significantly perturbed $2 \mathrm{~s}$ after an error.

Perturbation graphs (A, B) and a recovery plot (C) for 1:2 locking are shown in Figure 5] Note that the points occur in pairs, with small differences between the pairs of points leading to the appearance of oscillations in the recovery plot. These correspond to the pairs of postsynaptic spikes that occur between each pair of presynaptic spikes (two categories of cross intervals). As with 1 : 1 locking, all responses to errors for high precision input were identical (A); the maximum perturbation in this case being $19 \mathrm{~ms}$. Low precision (B) maximum perturbations ranged from about $17 \mathrm{~ms}$ to $20 \mathrm{~ms}$. The recovery time constant for $0.51 \leq e_{g} \leq 1.08$ was $\tau=0.27 \mathrm{~s}(\tau / I=1.19)$. Recovery slows down considerably after $e_{g}=1.2 \mathrm{~s}$, at about ten times the pre-error difference range.

As Figure 6(A) shows, $2: 3$ locking, unlike the previous lockings, exhibited some variability even in its high precision response to repeated errors - qualitatively similar to low precision simulations, though much smaller. Note the linear scale in graphs (A) and (B). The maximum high precision error perturbation was around $24 \mathrm{~ms}$ with a $1 \mathrm{~ms}$ spread; for low precision, this was $27 \mathrm{~ms}$ with a $5 \mathrm{~ms}$ spread (ignoring bimodality; see below). In both cases, there were triplets of clusters, corresponding to the three postsynaptic spikes occurring for each pair of presynaptic spikes (i.e., this was a period-three behavior). Differences among the three categories of cross intervals explain what appear to be oscillations in the recovery plot (C), as with $1: 2$ locking. Clusters had a bimodal distribution of perturbations, with some small (around 0-2ms) and some large. The recovery plot shows an initial peak at less than an order of magnitude more than the pre-error value, a slow recovery until around $e_{g}=2.5 \mathrm{~s}$; there were additional, lower peaks after $2.5 \mathrm{~s}$ (before eventual return to pre-error values, as indicated by the points before the error). The range of differences before the error $\left(e_{g}<0\right)$ was on the order of $1 \mathrm{~ms}$, compared to $2-5 \mu$ s for $1: 1$ and $1: 2$ lockings.

\subsection{Quasiperiodicities}

A quasiperiodic behavior is one in which the phase of the response shifts with respect to the stimulus, eventually taking on any value within some range (Ermentrout and Rinzel, 1984). This is made apparent by the phase return map in Figure 7(A), which is a one-dimensional curve covering the range $0-I$. Note that a phase of zero is equivalent to one of $I$, so the graph corresponds to a torus and the curve is not discontinuous. There was no apparent qualitative difference (and little quantitative difference) between high (B) and low (C) precision error responses; if anything, there was even a slight decrease in postsynaptic spike timing variability after an error. This is especially apparent in the recovery plot (D), with a maximum range of $I$ before an error and a minimum range (ignoring the high frequency "oscillations" every third spike - the "ghost" of the nearby $2: 3$ locking) of around $95 \mathrm{~ms}$ at $e_{g} \approx 1 \mathrm{~s}$.

Figure 8 presents results for a high-input-rate $(N / I>1)$ quasiperiodicity. As can be seen 

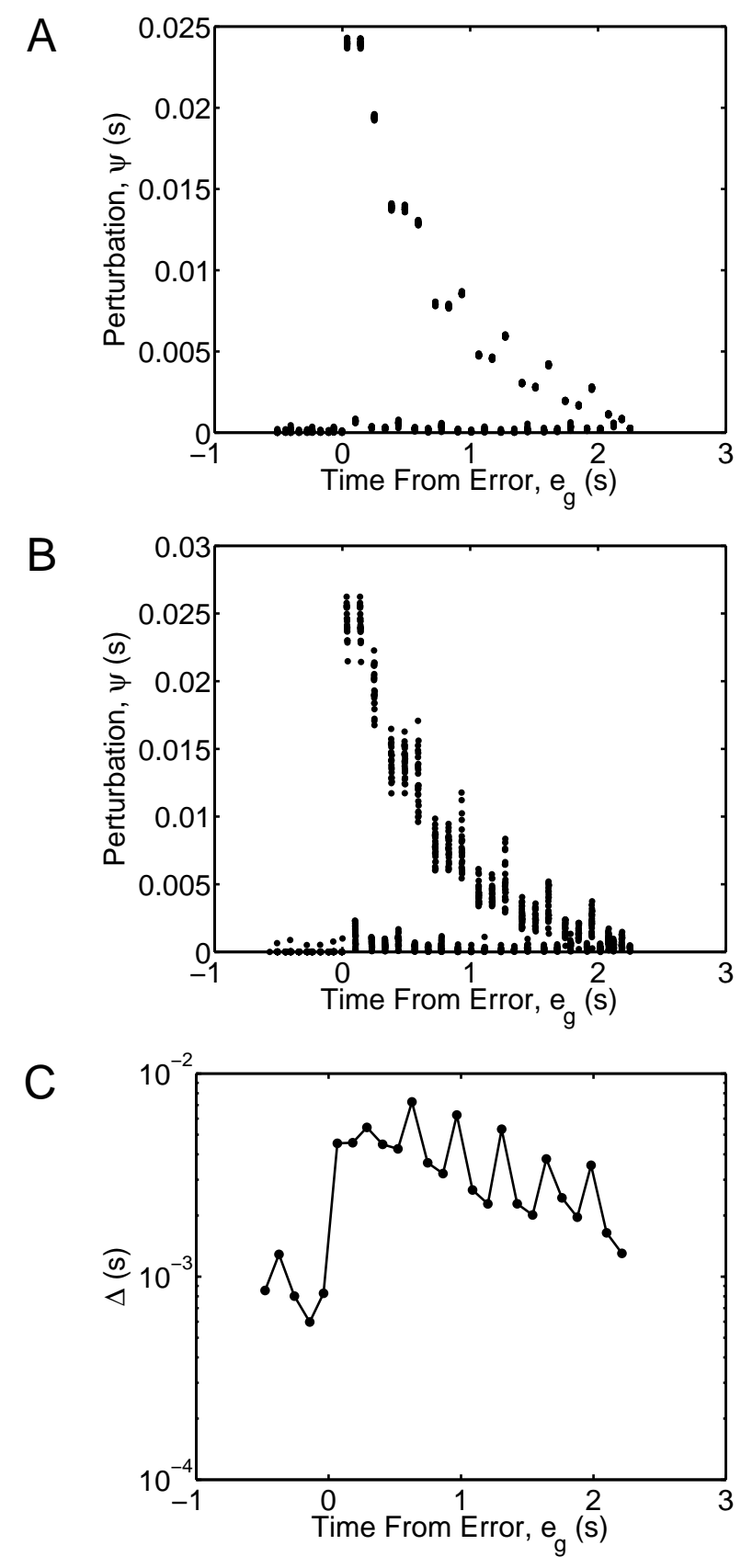

Figure 6: Results for $2: 3$ locking $(I=168.43 \mathrm{~ms}, N / I=0.62)$. Both high precision (A) and low precision (B) perturbation graphs show ranges of responses to different errors. Recovery plot (C) shows reduced, long-duration error aftereffect. ( $m=49$ errors, $c=25$ spikes/error)

in the phase return map (A), the model only fired within a relatively restricted range of phases, approximately 59-63ms after a presynaptic spike. This is an example of a windowed behavior (Segundo et al., 1991a), in which the postsynaptic cell is only able to fire within some narrow 

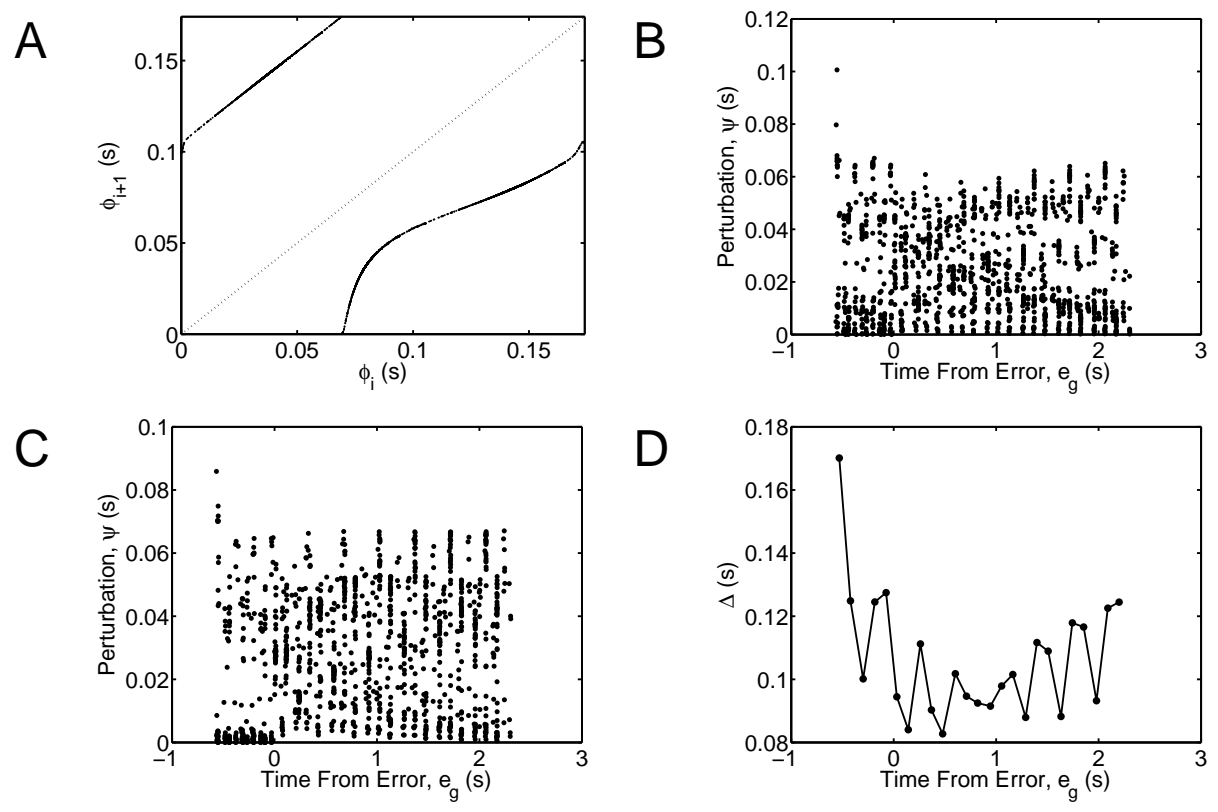

Figure 7: Error responses for quasiperiodic behavior near $2: 3$ locking $(I=174.04 \mathrm{~ms}, N / I=0.60)$. Phase return map (A) is a 1D curve. High precision (B) and low precision (C) perturbation plots. Note linear Y axis for recovery plot (D). ( $m=49$ errors, $c=25$ spikes/error)
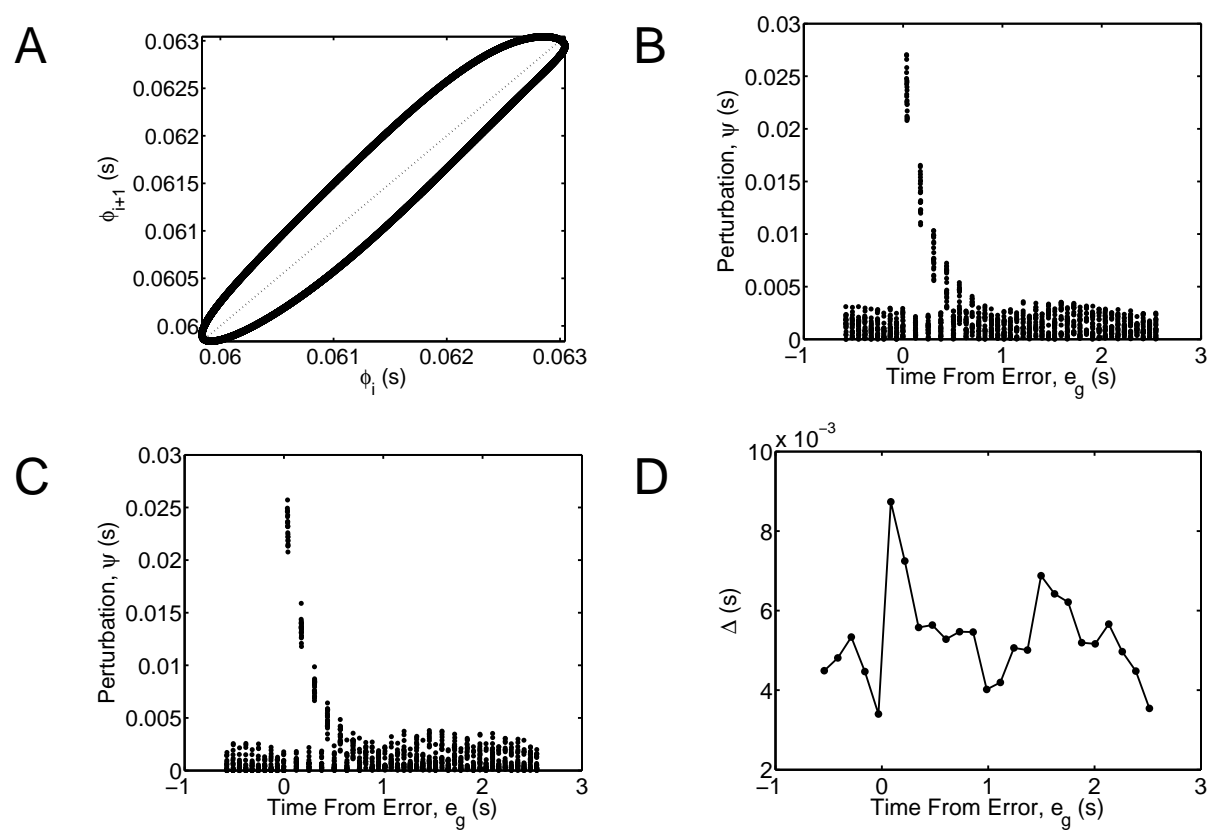

Figure 8: Error responses for windowed quasiperiodic behavior $(I=63.67 \mathrm{~ms}, N / I=1.64)$. Phase return map (A) is a $1 \mathrm{D}$ curve. High precision (B) and low precision (C) perturbation plots, Note linear $\mathrm{Y}$ axis for recovery plot $(\mathrm{D})$. ( $m=49$ errors, $c=25$ spikes/error) 
A

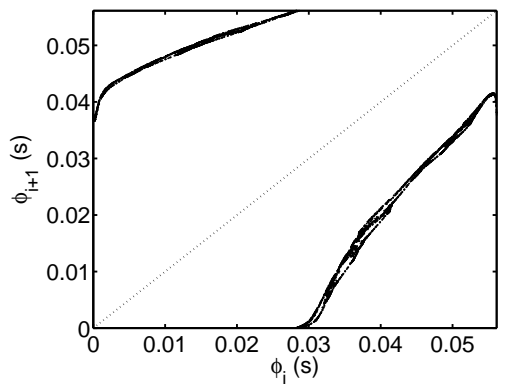

C

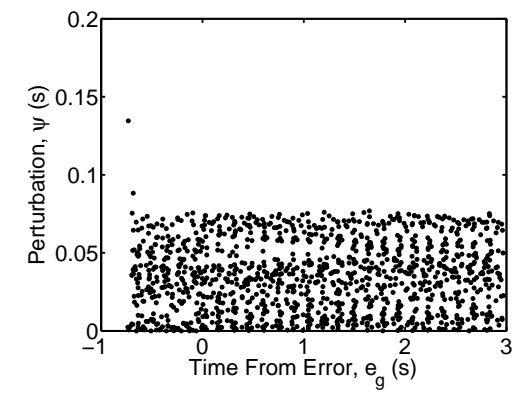

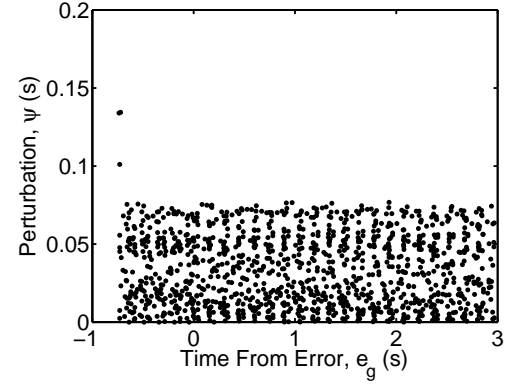

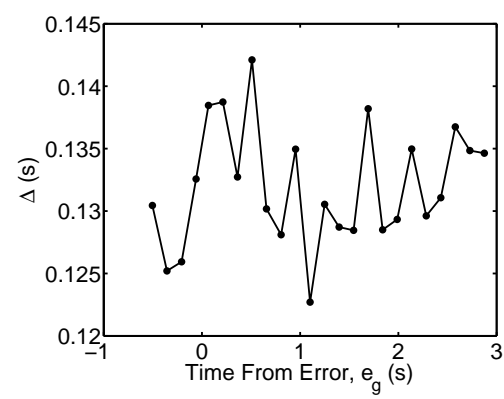

Figure 9: Chaotic behavior $(I=56.14 \mathrm{~ms}, N / I=1.86)$. Phase return map (A) is not a 1D curve. High precision (B) and low precision (C) perturbation plots. Note linear Y axis for recovery plot (D). ( $m=49$ errors, $c=25$ spikes/error)

time. Both high (B) and low (C) precision had a spike timing variability of around $3 \mathrm{~ms}$ before an error, with a peak perturbation of $28 \mathrm{~ms}$ and perturbation range of about $8 \mathrm{~ms}$ after an error. The range of differences (D) was less than doubled by an error, with an oscillatory recovery with period a bit over $1 \mathrm{~s}$ and apparent complete recovery after $4 \mathrm{~s}$.

\subsection{Chaos}

Figure 9 shows the error responses for a high-input-rate chaotic behavior. The phase return map (A) is "braided;" clearly not a 1D curve. The neuron could fire between 0 and $56 \mathrm{~ms}(I)$ after a presynaptic spike, with a postsynaptic interval range of $139-155 \mathrm{~ms}$ and mean of $148 \mathrm{~ms}$. So, this response was not windowed. As with the low-input-rate quasiperiodic behavior, there was little difference between high (B) and low (C) precision responses - in both cases, postsynaptic spike timing variability decreased after the error. There was perhaps a slight increase in the recovery plot (D) after an error, with subsequent decrease and increase again, but this was not dramatic.

\subsection{Bifurcation Boundaries}

The earlier locking examples were chosen to lie within the middle of the range of presynaptic rates that elicited the behavior. For input rates near an extreme presynaptic rate, different results were found. This is illustrated in Figure 10 for an input near the low rate bifurcation point bounding $1: 1$ locking. As in the middle of the range, high precision inputs (A) elicited the same response to 

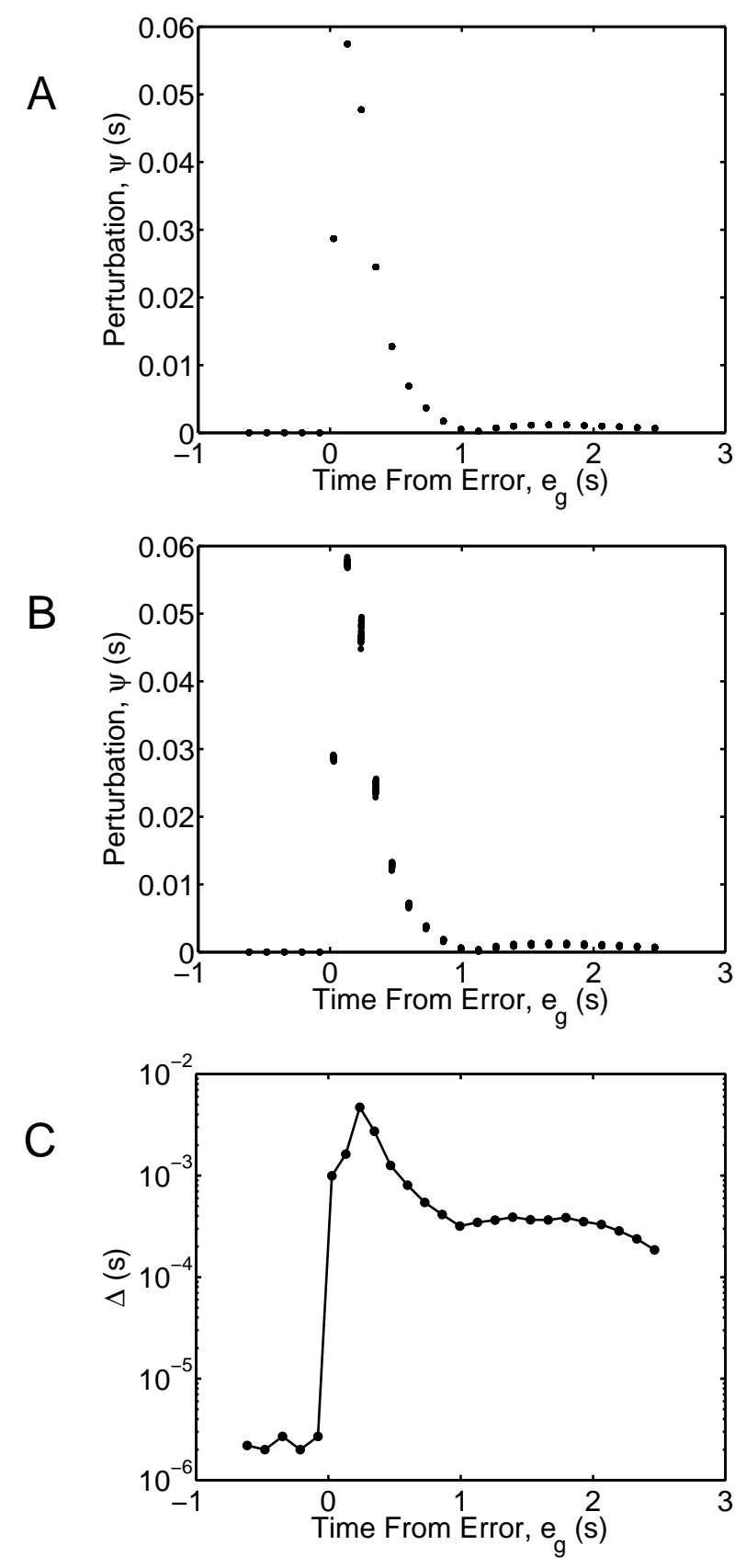

Figure 10: Results for edge of $1: 1$ locking $(I=133.88 \mathrm{~ms}, N / I=0.78)$. High precision perturbation graph (A) shows identical responses to all errors; low precision (B) shows range of responses. Both differ from farther away from the bifurcation (Figure 4). Recovery plot (C) shows greatly extended recovery. ( $m=49$ errors, $c=25$ spikes/error) 
every error, with a maximum perturbation of almost 60ms. For low precision inputs (B), maximum error perturbations were between $55 \mathrm{~ms}$ and $60 \mathrm{~ms}$. Unlike the previous $1: 1$ example, the peak perturbation was reached slowly - after three postsynaptic spikes - and the recovery was more gradual $(\mathrm{C})$, with a broad secondary peak around $e_{g}=1.5 \mathrm{~s}$ and a value still about two orders of magnitude greater than that before the error at $e_{g}=2.5$.

\section{Discussion}

This paper has examined the interaction of neuron dynamics and spike timing precision in error correction in a model of the living prototype of an inhibitory synapse and postsynaptic neuron. The dynamical behaviors included locked, quasiperiodic, and chaotic; additional issues, such as proximity to bifurcation points and windowing, were touched upon. All analysis was based on spike times and interspike intervals, rather than state space analysis. This is especially significant, in the author's view, because that is exactly the message that neurons transmit to each other. Thus, variations seen here are at least potentially significant (as opposed to changes in internal state that might have no impact on spike timing).

It is important to remember that, in all cases, high precision erroneous simulations were compared to high precision error free ones and low precision erroneous simulations were compared to low precision error free. Thus, when a recovery plot was generated, values larger than those just before an error showed that at least some low precision responses were significantly farther away from "full recovery" than the high precision ones.

Among all behaviors examined, phase lockings produced the most rapid recovery, with lower ratios that occur in broader rate domains $(1: 1$ and $1: 2)$ recovering faster. One reason for this likely was the gradient of the attractor basin surrounding such behaviors. During the acceptably exponential phase of their recoveries (roughly from $0.5 \mathrm{~s}$ to $1 \mathrm{~s}$ after an error), $1: 1$ and $1: 2$ locking had similar time constant $(0.20 \mathrm{~s}$ and $0.27 \mathrm{~s})$, despite the fact that their associated presynaptic rates differed by more than a factor of two. The conclusion is that, at least for that phase of error recovery, it was not the case that each presynaptic spike simply corrected the error by some amount proportional to the perturbation. Rather, the gradients of the two basins of attraction - at least at those distances from the attractors - were similar. Based on computed recovery time constants, the worst case low precision recoveries had significantly longer recovery times - on the order of seconds longer.

For 2 : 3 locking and all nonlocked responses, the "excess perturbation" of low precision versus high precision (the peak of the recovery plot) was much less than for the lower ratio locked behaviors. In fact, for $2: 3$ locking, it was roughly a factor of five greater, while for all nonlocked behaviors it was less than a factor of two. There were two possible mechanisms underlying this: a narrow rate domain that elicited the particular behavior and desynchronization of erroneous and error free responses by errors.

The first matter, that of domain width, was especially relevant to $2: 3$ locking. As can be seen

by comparing Figure 6 (C) to Figures 4(C) and 5(C), the peak was not too different than the lower ratio lockings; the difference was the degree of perturbation before the error (more than two orders of magnitude higher in the case of $2: 3$ locking, roughly at the level of the added low precision 
jitter). This was probably caused by the jitter in the low precision simulations bumping the state out of the locking behavior (Beierholm et al., 2001, Tiesinga and Sejnowski, 2001), causing the two low precision simulations to remain partially desynchronized - they were, at least part of the time, away from the synchronizing effect of the periodic attractor (see discussion below of bifurcation boundary transitions).

That was a "minor" desynchronization effect, because there was a periodic attractor which tended to pull the erroneous and error free simulations' states back together. Such was not the case for aperiodic behaviors. In those cases (quasiperiodicity and chaos), once the neuron had been perturbed by the first error, returning to the attractor did not bring its subsequent spikes back to the same times they would have occurred in the absence of the error. This was the case for both high and low precision. For the quasiperiodic cases (Figures 7 and [8), there were "preferred" firing intervals (where the phase return map was closest to the diagonal in both figures, and additionally due to windowing itself for windowed quasiperiodicity). The closer the quasiperiodicity came to being periodic (the more dominant the preferred firing intervals were), the more it would act to resynchronize the erroneous simulation with the error free one.

For Figure 7, this was not very close at all (about 25ms), the erroneous and error free experiments were completely desynchronized by the first error, and as a result their "perturbations" in Figures 7(B) and (C) before the error could be as large as the full range of possible phase differences $(I / 2)$. Additionally, the high and low precision cases thereafter were also decorrelated, and so the range of their perturbation differences in (D) was the sum of their maxima. However, in both the high and low perturbation cases, errors arrived at approximately the same time (within $\pm w I$ of each other), and so the errors paradoxically served as a partial resynchronizing input, as indicated by the decrease in (D) after the error.

Windowed quasiperiodicity, however, behaved more like a weak locking. As Figure 8 shows, the neuron was constrained to fire within a $4 \mathrm{~ms}$ window relative to an input spike when stationary (A), which was much less than the $25-30 \mathrm{~ms}$ perturbation induced by an error $(\mathrm{B}, \mathrm{C})$. As a result, the cell exhibited an oscillatory recovery, with high precision input being somewhat more effective (D).

Chaos, by definition of its hallmark sensitive dependence on initial conditions, was the extreme case of these desynchronizing processes, with the jitter of low precision leading those cases to diverge rapidly from high precision and the presence of errors having a similar effect between erroneous and error free experiments. As Figure 9(D) shows, error aftereffects were qualitatively similar to (though perhaps more extreme than) that for quasiperiodicity in Figure 7(D), with the similarly-timed errors affecting the high and low precision erroneous experiments in similar fashion.

The final results in Figure 10 address the issue of proximity to a bifurcation boundary. This is also relevant to the effects of domain width and similarity or dissimilarity of attractor basins and attractors on error recovery. In both the high and low precision cases, in the absence of errors the neuron's state was on or near a period-one attractor at all times (the jitter was not large enough to destroy locking, as was verified by examination of the phase return map for the low precision case). Unlike the simulation in Figure 4, however, the perturbation produced by an error did not displace the state into the basin of another $1: 1$ locking. Instead, the perturbation moved the state 
across the bifurcation boundary into the basins of qualitatively different attractors, and its motion thereafter was very different than that of recovery exclusively through period-one attractor basins.

This raises an important point regarding the nature of the perturbation produced by an error. Because the presynaptic intervals served as a control parameter, errors were not merely perturbations of the neuron's state within its state space, $\Sigma \subset \mathbb{R}^{n}$. Instead, they were perturbations within the system's response space, $I \times \Sigma$ (Thom, 1975, Abraham and Shaw, 1984). The perturbation of an ongoing behavior near a bifurcation boundary or within a narrow domain (such as in Figures 6 10) displaced the neuron's state across domains of different behaviors. After the perturbation, the re-established sequence of spike times drew the neuron through its response space and back across (possibly multiple) bifurcations into basins of attractors qualitatively like its "home" (stationary attractor). Its evolution, however, because of these dissimilar basins, didn't necessarily lead it toward the region of $\Sigma$ that contained this home. As a result, the perturbation and recovery plots could show multimodal, or oscillatory, shapes.

Having established that even a small amount of spike timing jitter can have observable effects on the time course of error recovery (and potentially on the activity of further postsynaptic neurons), subsequent work is progressing along a number of fronts:

- A more complete model of the bifurcation behavior of error recovery (Stiber and Holderman, 2004a) is being developed. Rather than using some property of the stationary behavior of a dynamical system, one can examine how properties of transient neural responses depend on pre-existing stationary behaviors.

- The role of degree of spike timing accuracy on error is being investigated (Stiber and Holderman, 2004b). The current results show that even small amounts of jitter can have a significant effect on error recovery. The amounts used here were in the range of $1-4 \mathrm{~ms}$ or so, and thus are relevant to a range of biological systems, based on observations (for multiple spike trains) of correlations and correlation-dependent activity in retinal ganglion cells and lateral geniculate cells, and are at the low end for some cortical cells (Bair, 1999). Preliminarily, order of magnitude jitter increases in general produce similar effects, though, as might be expected in a nonlinear system, linear increases do not appear to produce linear changes in recovery.

- The transient response of this model is being examined in terms of its internal state variables (Stiber and Pottorf, 2004), as a preliminary to developing a simplified model that captures the error responses and is more amenable to analytic approaches. Early results suggest that spike rate adaptation is the most significant contributor to the observed error responses.

A final matter is that of the application of Shannon's information theory to coding by nonlinear dynamical neurons. Techniques from the field of information theory (Shannon, 1948, Cover and Thomas, 1991) are currently of great interest in addressing the question of what spike trains can be said to carry identical information (Bialek et al., 1991; Borst and Theunissen, 1999; Fuhrmann et al., 2002), and thus whether the timing of individual spikes or average firing rate is relevant. Entropy calculations can be quantified in terms of bits of information per spike - a compelling statistic. The typical experimental approach is to present a stimulus multiple times and use the "average" response (where this averaging might be accomplished in varying ways) to compute entropy or mutual information. This is then used to infer characteristics of the neural code (e.g., rate versus time codes). 
It is important, however, to remember that information theory tells one nothing about the semantics of a code (Shannon and Weaver, 1949). Primarily, and in conjunction with knowledge of the noise in a communication channel, it addresses issues of information capacity: how close to optimally compressed a signal is. This part of Shannon's work - the Noisy Channel Coding Theorem - is usually used in neuroscience only in terms of estimating "channel noise" from multiple presentations of the same stimulus. However, another application of this theorem is the creation of codes that use more bits (symbols, or in the context of neural coding, greater spike timing precision) than the required minimum to provide for error correction in the presence of channel noise.

As these results clearly show, both the quantitative and qualitative results of a presynaptic error depend critically on a neuron's internal state's ongoing evolution. It is a mapping $f: I \times \Sigma \rightarrow O \times \Sigma$, from input and state to output and new state. This suggests that the computation performed by a neuron will inevitably result in a decrease in mutual information between its input and output, according to the Data Processing Theorem (Shannon, 1948, Cover and Thomas, 1991). In contrast, Shannon's formal definition of an encoder or decoder is a stationary mapping $f: A^{*} \rightarrow B^{*}$ from input sequences of symbols taken from some set $A$ to output sequences of symbols from some (possibly different) set $B$. In other words, Shannon's information theory applies when the encoding of an input symbol depends only on its context within the sequence of input symbols. On the other hand, for a nonlinear dynamical system, output depends on input and state, where state is not merely a function of input but also of intrinsic dynamics. Therefore, Shannon's information theory is conceptually incompatible with neural information processing. In practical terms, this implies that information theoretic techniques can only be used to establish lower bounds on matters such as spike timing precision.

\section{A SAO Model Equations}

The SAO model equations used here are documented more completely in (Edman et al., 1987; Stiber et al., 1997); the following is a brief summary. It is a permeability-based, rather than conductance-based, model with the following main equations:

$$
\begin{aligned}
\mathrm{d} V_{m} / \mathrm{d} t & =-\left(I_{N a}+I_{K}+I_{L, N a}+I_{L, K}+I_{L, C l}+I_{p}+I_{\text {bias }}+I_{s y n}\right) / C_{m} \\
I_{N a} & =A \bar{P}_{N a} m^{2} h l \frac{V_{m} F^{2}}{R T} \frac{\left[\mathrm{Na}^{+}\right]_{o}-\left[\mathrm{Na}^{+}\right]_{i} \exp F V_{m} / R T}{1-\exp F V_{m} / R T} \\
I_{K} & =A \bar{P}_{K} n^{2} r \frac{V_{m} F^{2}}{R T} \frac{\left[\mathrm{K}^{+}\right]_{o}-\left[\mathrm{K}^{+}\right]_{i} \exp F V_{m} / R T}{1-\exp F V_{m} / R T} \\
I_{L, X} & =A P_{L, X} \frac{V_{m} F^{2}}{R T} \frac{[X]_{o}-[X]_{i} \exp \left(F V_{m} / R T\right)}{1-\exp \left(F V_{m} / R T\right)} \\
I_{p} & =\frac{A F}{3} \frac{\bar{J}_{p, N a}}{\left(1+\frac{K_{m}}{\left[\mathrm{Na}^{+}\right]_{i}}\right)^{3}}
\end{aligned}
$$




$$
\begin{aligned}
I_{s y n}= & A P_{s y n} \frac{V_{m} F^{2}}{R T} \frac{\left[\mathrm{Cl}^{-}\right]_{o}-\left[\mathrm{Cl}^{-}\right]_{i} \exp \left(-F V_{m} / R T\right)}{1-\exp \left(-F V_{m} / R T\right)} \\
& \times \sum_{\forall s_{k} \leq t}\left(e^{\left(s_{k}-t\right) / \tau_{+}}-e^{\left(s_{k}-t\right) / \tau_{-}}\right)
\end{aligned}
$$

Table 1: Constants used in this simulation, in rough order of

\begin{tabular}{|c|c|c|}
\hline Constant & Value & Units (MKS) \\
\hline$C_{m}$ & $7.5 \times 10^{-9}$ & Farads \\
\hline$I_{\text {bias }}$ & $-2.5 \times 10^{-9}$ & A \\
\hline$A$ & $1.0 \times 10^{-7}$ & $\mathrm{~m}^{2}$ \\
\hline $\bar{P}_{N a}$ & $6.0 \times 10^{-6}$ & $\mathrm{~m} / \mathrm{s}$ \\
\hline$\left[\mathrm{Na}^{+}\right]_{o}$ & 325.0 & $\mathrm{mM}$ \\
\hline$\left[\mathrm{Na}^{+}\right]_{i}$ & 10.0 & $\mathrm{mM}$ \\
\hline$T$ & 291.15 & ${ }^{\circ} \mathrm{K}$ \\
\hline $\bar{P}_{K}$ & $2.0 \times 10^{-6}$ & $\mathrm{~m} / \mathrm{s}$ \\
\hline$\left[\mathrm{K}^{+}\right]_{o}$ & 5.0 & $\mathrm{mM}$ \\
\hline$P_{L, N a}$ & $5.0 \times 10^{-10}$ & $\mathrm{~m} / \mathrm{s}$ \\
\hline$P_{L, K}$ & $1.8 \times 10^{-8}$ & $\mathrm{~m} / \mathrm{s}$ \\
\hline$P_{L, C l}$ & $1.1 \times 10^{-9}$ & $\mathrm{~m} / \mathrm{s}$ \\
\hline$\left[\mathrm{Cl}^{-}\right]_{o}$ & 650.0 & $\mathrm{mM}$ \\
\hline$\left[\mathrm{Cl}^{-}\right]_{i}$ & 46.0 & $\mathrm{mM}$ \\
\hline $\bar{J}_{p, N a}$ & $6.0 \times 10^{-6}$ & $\mathrm{~mol} /\left(\mathrm{m}^{2} \mathrm{~s}\right)$ \\
\hline$K_{m}$ & 13.4 & $\mathrm{mM}$ \\
\hline$P_{s y n}$ & $6.0 \times 10^{-8}$ & $\mathrm{~m} / \mathrm{s}$ \\
\hline$\tau_{+}$ & $0.25 \times 10^{-3}$ & $\mathrm{~s}$ \\
\hline$\tau_{-}$ & $0.5 \times 10^{-3}$ & $\mathrm{~s}$ \\
\hline$v_{m}$ & 0.0 & dimensionless \\
\hline$v_{h}$ & 0.0 & dimensionless \\
\hline$v_{l}$ & 0.0 & dimensionless \\
\hline$v_{n}$ & 0.03 & dimensionless \\
\hline$v_{r}$ & 0.5 & dimensionless \\
\hline$z_{m}$ & -3.1 & dimensionless \\
\hline$z_{h}$ & 4.0 & dimensionless \\
\hline$z_{l}$ & 3.5 & dimensionless \\
\hline$z_{n}$ & -2.6 & dimensionless \\
\hline$z_{r}$ & 4.0 & dimensionless \\
\hline$V_{m}$ & $-19.0 \times 10^{-3}$ & V \\
\hline$V_{h}$ & $-35.0 \times 10^{-3}$ & V \\
\hline$V_{l}$ & $-53.0 \times 10^{-3}$ & V \\
\hline$V_{n}$ & $-18.0 \times 10^{-3}$ & V \\
\hline
\end{tabular}
appearance in equations in this appendix. 
Table 1: Constants used in this simulation, cont'd. Note that the $V_{m}$ here is the voltage associated with the $m$ gating variable, not the membrane potential.

\begin{tabular}{|l|r|l|}
\hline Constant & Value & Units (MKS) \\
\hline$V_{r}$ & $-56.0 \times 10^{-3}$ & $\mathrm{~V}$ \\
$\bar{\tau}_{m}$ & $0.3 \times 10^{-3}$ & $\mathrm{~s}$ \\
$\bar{\tau}_{h}$ & $5.0 \times 10^{-3}$ & $\mathrm{~s}$ \\
$\bar{\tau}_{l}$ & $1700.0 \times 10^{-3}$ & $\mathrm{~s}$ \\
$\bar{\tau}_{n}$ & $6.0 \times 10^{-3}$ & $\mathrm{~s}$ \\
$\bar{\tau}_{r}$ & $2000.0 \times 10^{-3}$ & $\mathrm{~s}$ \\
\hline
\end{tabular}

Values for constants are given in Table 1 Besides active and leak $\mathrm{Na}^{+}, \mathrm{K}^{+}$, and $\mathrm{Cl}^{-}$currents, there is a bias current $\left(I_{\text {bias }}\right)$, generated in response to muscle stretch, an active pump $\left(I_{p}\right)$, and a synaptic current $\left(I_{s y n}\right)$. There are five gating variables, $m, h, l, n$, and $r$, with first-order kinetics described by (7) (where $g \in\{m, h, l, n, r\}$ ).

$$
\begin{aligned}
\mathrm{d} g / \mathrm{d} t & =\left(g_{\infty}-g\right) \frac{1}{\tau_{g}} \\
g_{\infty} & =v_{g}+\frac{1-v_{g}}{1+\exp \left[\frac{z_{g} e}{k T}\left(V_{m}-V_{g}\right)\right]} \\
\tau_{g} & =\frac{Q_{g} \bar{\tau}_{g}}{\exp \left[\frac{\delta_{g} z_{g} e}{k T}\left(V_{m}-V_{g}\right)\right]+\exp \left[\frac{\left(\delta_{g}-1\right) z_{g} e}{k T}\left(V_{m}-V_{g}\right)\right]} \\
Q_{g} & =\left(\frac{1-\delta_{g}}{\delta_{g}}\right)^{\delta_{g}}+\left(\frac{1-\delta_{g}}{\delta_{g}}\right)^{\delta_{g}-1}
\end{aligned}
$$

\section{References}

Abraham, R. and Shaw, C. (1984). Dynamics - The Geometry of Behavior, volume 1-4. Aerial Press, Santa Cruz, Calif.

Allen, C. and Stevens, C. (1994). An evaluation of causes for unreliability of synaptic transmision. Proc. Natl. Acad. Sci. USA, 91:10380-3.

Bair, W. (1999). Spike timing in the mammalian visual system. Curr. Opin. Neurobiol., 9(4):44753.

Beierholm, U., Nielsen, C. D., Ryge, J., Alstrøm, P., and Kiehn, O. (2001). Characterization of reliability of spike timing in spinal interneurons during oscillating inputs. J. Neurophysiol., 86:1858-68. 
Bialek, W., Rieke, F., de Ruyter van Steveninck, R. R., and Warland, D. (1991). Reading a neural code. Science, 252:1854-7.

Borst, A. and Theunissen, F. (1999). Information theory and neural coding. Nature Neurosci., 2(11):947-57.

Bryant, H., Marcos, A. R., and Segundo, J. (1973). Correlations of neuronal spike discharges produced by monosynaptic connections and by common inputs. J. Neurophysiol., 36:205-25.

Buño, W., Fuentes, J., and Barrio, L. (1987). Modulation of pacemaker activity by IPSP and brief length perturbations in the crayfish stretch receptor. J. Neurophysiol., 57:819-34.

Cover, T. and Thomas, J. (1991). Elements of Information Theory. John Wiley and Sons, Inc.

Cox, D. and Isham, V. (1980). Point Processes. Chapman and Hall, London.

Dan, Y., Alonso, J.-M., Usrey, W. M., and Reid, R. C. (1998). Coding of visual information by precisely correlated spikes in the lateral geniculate nucleus. Nature Neurosci., 1(6):501-7.

Edman, A., Gestrelius, S., and Grampp, W. (1987). Analysis of gated membrane currents and mechanisms of firing control in the rapidly adapting lobster stretch receptor neurone. $J$. Physiol., 384:649-69.

Ermentrout, G. and Rinzel, J. (1984). Beyond a pacemaker's entrainment limit: Phase walkthrough. Am. J. Physiol., 246:R102-6.

Fuhrmann, G., Segev, I., Markram, H., and Tsodyks, M. (2002). Coding of temporal information by activity-dependent synapses. J. Neurophysiol., 87:140-8.

Glass, L. and Mackey, M. (1988). From Clocks to Chaos. Princeton University Press.

Hindmarsh, A. (1983). ODEPACK: A systematized collection of ODE solvers. In Stepleman, R., editor, Scientific Computing, pages 55-64. North-Holland, Amsterdam.

Katz, B. (1966). Nerve, Muscle, and Synapse. McGraw-Hill, New York.

Kohn, A., da Rocha, A., and Segundo, J. (1981). Presynaptic irregularity and pacemaker inhibition. Biol. Cybern., 41:5-18.

Kohn, A. and Segundo, J. (1983). Neuromime and computer simulations of synaptic interactions between pacemakers. Mathematical expansions of existing models. J. Theoret. Neurobiol., 2:101-25.

Lestienne, R. (2001). Spike timing, synchronization and information processing on the sensory side of the central nervous system. Prog. Neurobiol., 65:545-591.

Maršálek, P., Koch, C., and Maunsell, J. (1997). On the relationship between synaptic input and spike output jitter in individual neurons. Proc. Natl. Acad. Sci. USA, 94:735-40. 
Rapp, P., Zimmerman, I., Albano, A., deGuzman, G., and Greenbaun, N. (1985). Dynamics of spontaneous neural activity in the simian motor cortex: The dimension of chaotic neurons. Phys. Lett. A, 110:335-8.

Ruelle, D. (1989). Elements of Differentiable Dynamics and Bifurcation Theory. Academic Press, Boston.

Segundo, J. (1979). Pacemaker synaptic interactions: modeled locking and paradoxical features. Biol. Cybern., 35:55-62.

Segundo, J., Moore, G., Stensaas, L., and Bullock, T. (1963). Sensitivity of neurones in aplysia to temporal pattern of arriving impulses. J. Exp. Biol., 40:643-67.

Segundo, J., Perkel, D., Wyman, H., Hegstad, H., and Moore, G. (1968). Input-output relations in computer-simulated nerve cells. Influence of the statistical properties, strength, number, and inter-dependence of excitatory pre-synaptic terminals. Kybernetic, 4:157-71.

Segundo, J., Stiber, M., Altshuler, E., and Vibert, J.-F. (1994a). Transients in the inhibitory driving of neurons and their post-synaptic consequences. Neurosci., 62(2):459-80.

Segundo, J., Stiber, M., Vibert, J.-F., and Hanneton, S. (1995a). Periodically modulated inhibition and its post-synaptic consequences. II. Influence of pre-synaptic slope, depth, range, noise and of post-synaptic natural discharges. Neurosci., 68(3):693-719.

Segundo, J., Tolkunov, B., and Wolfe, G. (1976). Relation between trains of action potentials across an inhibitory synapse. Influence of presynaptic irregularity. Biol. Cybern., 24:169-79.

Segundo, J., Vibert, J.-F., Pakdaman, K., Stiber, M., and Diez Martinez, O. (1994b). Noise and the neurosciences: a long history, a recent revival and some theory. In Pribram, K., editor, Origins: Brain and Self Organization. Lawrence Erlbaum Assoc.

Segundo, J., Vibert, J.-F., and Stiber, M. (1998). Periodically modulated inhibition of living pacemaker neurons. III. The heterogeneity of the postsynaptic spike trains and how control parameters affect it. Neurosci., 87(1):15-47.

Segundo, J., Vibert, J.-F., Stiber, M., and Hanneton, S. (1993). Synaptic coding of periodically modulated spike trains. In Proc. ICNN, pages 58-63, San Francisco.

Segundo, J., Vibert, J.-F., Stiber, M., and Hanneton, S. (1995b). Periodically modulated inhibition and its post-synaptic consequences. I. General features. Influences of modulation frequency. Neurosci., 68(3):657-92.

Segundo, J. P., Altshuler, E., Stiber, M., and Garfinkel, A. (1991a). Periodic inhibition of living pacemaker neurons: I. Locked, intermittent, messy, and hopping behaviors. Int. J. Bifurcation and Chaos, 1(3):549-81. 
Segundo, J. P., Altshuler, E., Stiber, M., and Garfinkel, A. (1991b). Periodic inhibition of living pacemaker neurons: II. Influences of driver rates and transients and of non-driven postsynaptic rates. Int. J. Bifurcation and Chaos, 1(4):873-90.

Shannon, C. E. (1948). A mathematical theory of communication. Bell System Technical Journal, 27:379-423, 623-56.

Shannon, C. E. and Weaver, W. (1949). The Mathematical Theory of Communication. University of Illinois Press, Urbana, Illinois.

Stiber, M. (2003). Non-information-maximizing neural coding. In Proc. IJCNN'03, Portland.

Stiber, M. and Holderman, T. (2004a). Global behavior of neural error correction. In Proc. IJCNN, Budapest, Hungary.

Stiber, M. and Holderman, T. (2004b). Stochastic-resonance-like effects in neural error correction. in preparation.

Stiber, M., Ieong, R., and Segundo, J. (1997). Responses to transients in living and simulated neurons. IEEE Trans. Neural Networks, 8(6):1379-85.

Stiber, M., Pakdaman, K., Vibert, J.-F., Boussard, E., Segundo, J., Nomura, T., Sato, S., and Doi, S. (1996). Complex responses of living pacemaker neurons to pacemaker inhibition: a comparison of dynamical models. Biosystems, 40:177-88.

Stiber, M. and Pottorf, M. (2004). Response space construction for neural error correction. In Proc. IJCNN, Budapest, Hungary.

Stiber, M. and Segundo, J. P. (1993). Dynamics of synaptic transfer in living and simulated neurons. In Proc. ICNN, pages 75-80, San Francisco.

The MathWorks, Inc. http://www.mathworks.com/.

Thom, R. (1975). Structural Stability and Morphogenesis. W.A. Benjamin, Inc., Reading, MA.

Tiesinga, P. (2002). Precision and reliability of periodically and quasiperiodically driven integrateand-fire neurons. Phys. Rev. E, 65(4):041913.

Tiesinga, P. and Sejnowski, T. J. (2001). Precision of pulse-coupled networks of integrate-and-fire neurons. Network: Comput. Neural Syst., 12:215-33.

Winfree, A. (1980). The Geometry of Biological Time. Springer-Verlag, New York. 